\title{
TRACE-CLASS OPERATORS AND FOURIER TRANSFORM
}

\author{
YAOGAN MENSAH ${ }^{1,2, *}$ \\ ${ }^{1}$ Department of Mathematics, University of Lomé, Togo \\ ${ }^{2}$ ICMPA, University of Abomey-Calavi, Benin \\ *Corresponding author: mensahyaogan2@gmail.com
}

Received Apr. 19, 2021

\begin{abstract}
Aвstract. A weighted Lebesgue-Banach convolution algebra is constructed. On this algebra, a Fourier transform related to trace-class operators is defined. Convergence properties are obtained. Also a Fourier transform of a system, modeled by an operator, is defined and basic properties are studied.
\end{abstract}

2010 Mathematics Subject Classification. 42B10; 43A32; 47F99.

Key words and phrases. trace-class operator; Fourier transform; convolution; weight; system.

\section{InTRODUCTION}

The importance of the Fourier transform is no longer to be proved. It plays a major role in several areas of modern mathematics. It has many generalizations in various directions; see for instance $[1,2,8]$. This paper is a contribution to the generalization of the Fourier transform. The Fourier transform in the non-commutative setting interplays a lot with tools from operator theory. For instance, the Fourier transform of a complex valued function on a locally compact group evaluated at a unitary irreducible representation $\sigma$ turns out to be a bounded operator on the representation space of $\sigma[4]$.

Let $\mathfrak{H}$ be a separable Hilbert space. It is known that the Schatten space $B(\mathfrak{H})$ of all bounded operators on $\mathfrak{H}$ is the topological dual of the Schatten space $B_{1}(\mathfrak{H})$ of all trace-class operators on $\mathfrak{H}$ when each of them is endowed with a natural norm [9]. From this duality, we defined a Fourier transform of functions of a weighted Lebesgue space and we derive some properties. The rest of the paper is organized as follows. In section 2, we recall definitions and basic

DOI: 10.28924/APJM/8-21

()2021 Asia Pacific Journal of Mathematics 
facts involving trace-class operators. Section 3 is devoted to the introduction of a weighted convolution algebra related to a representation of a locally compact group; a convolution result is proved here. In section 4, we introduced a Fourier transform formula. We show that it generalizes the classical Fourier transform formula. We also proved convergence results. Finally, in section 5, we defined the Fourier transform of a system and derived some basic properties.

\section{Trace-Class operators}

Throughout this paper, the word operator means linear operator. In this section, we recall mainly what is a trace-class operator on a Hilbert space. Let $\mathfrak{H}$ be a complex separable Hilbert space. An operator $T: \mathfrak{H} \longrightarrow \mathfrak{H}$ is said to be bounded if

$$
\sup \{\|T x\|:\|x\| \leq 1\}<\infty
$$

We denote by $B(\mathfrak{H})$ the Banach space of all bounded operators on $\mathfrak{H}$ endowed with the norm

$$
\|T\|=\sup \{\|T x\|:\|x\| \leq 1\} .
$$

An operator $T: \mathfrak{H} \longrightarrow \mathfrak{H}$ is said to be positive if $\langle T x, x\rangle \geq 0$ for all $x \in \mathfrak{H}$. A bounded positive operator $T$ is said to be a trace-class operator if $\sum_{n}\left\langle T e_{n}, e_{n}\right\rangle<\infty$ for any orthonormal basis $\left(e_{n}\right)$ of $\mathfrak{H}$. An arbitrary bounded operator is said to be trace-class if the positive operator $|T|:=\sqrt{T^{*} T}$ is trace-class $\left[5\right.$, page 11]. We denote by $B_{1}(\mathfrak{H})$ the space of all trace-class operators on the Hilbert space $\mathfrak{H}$. The trace-norm on $B_{1}(\mathfrak{H})$ is defined by

$$
\|T\|_{1}=\operatorname{tr}(|T|)=\sum_{n}\left\langle|T| e_{n}, e_{n}\right\rangle
$$

If $\mathfrak{H}$ is of finite dimension, then every operator on $\mathfrak{H}$ is a trace-class operator and the trace coincides with the usual trace of a matrix. In the theory of von Neumann ideals, the duality

$$
\left(B_{1}(\mathfrak{H})\right)^{*}=B(\mathfrak{H})
$$

is well-known [9, page 77]. Our formulation of the Fourier transformation will be based on the above fact (see Section 4 ). 


\section{Weighted CONVOLUtion ALGEBRA AND Fourier tRANSFORM}

Let $G$ be a locally compact group. We consider a continuous representation $\sigma$ of $G$ on a complex Hilbert space $\mathfrak{H}_{\sigma}$. Then for each $x \in G, \sigma(x)$ is a bounded operator on $\mathfrak{H}_{\sigma}$. Set

$$
\omega_{\sigma}(x)=\|\sigma(x)\|, x \in G .
$$

As such, we define a function $\omega_{\sigma}$ from $G$ into the set of nonnegative reals $\mathbb{R}_{+}$, see $[10$, page, 241].

Theorem 3.1. The function $\omega_{\sigma}$ is a (continuous) submultiplicative weight on $G$.

Proof. The function $\omega_{\sigma}$ inherits its continuity from that of $\sigma$ and from the automatic continuity of the norm. Now, let $x, y \in G$.

$$
\begin{aligned}
\omega_{\sigma}(x y) & =\|\sigma(x) \sigma(y)\| \\
& \left.\leq\|\sigma(x)\|\|\sigma(y)\| \quad \text { (Banach algebra property of } B\left(\mathfrak{H}_{\sigma}\right)\right) \\
& \leq \omega_{\sigma}(x) \omega_{\sigma}(y) .
\end{aligned}
$$

We denote by $L_{\omega_{\sigma}}^{1}(G)$ the weighted Lebesgue space defined by the weight $\omega_{\sigma}$. As a set, $L_{\omega_{\sigma}}^{1}(G)$ contains complex Borel measurable (class of) functions $f$ on $G$ such that

$$
\int_{G}|f(x)| \omega_{\sigma}(x) d x<\infty .
$$

Here the integration is taking against a left Haar measure of $G$. The norm on $L_{\omega_{\sigma}}^{1}(G)$ is given by

$$
\|f\|_{L_{\omega_{\sigma}}^{1}}=\int_{G}|f(x)| \omega_{\sigma}(x) d x .
$$

We recall that for $f, g \in L_{\omega_{\sigma}}^{1}(G)$ the convolution $f * g$ is defined by

$$
f * g(x)=\int_{G} f\left(x y^{-1}\right) g(y) d y .
$$

Theorem 3.2. Let $G$ be a locally compact group. Let $\sigma$ be a representation of $G$. Then

$$
\|f * g\|_{L_{\omega_{\sigma}}^{1}} \leq\|f\|_{L_{\omega_{\sigma}}^{1}}\|\check{\Delta} g\|_{L_{\omega_{\sigma}}^{1}}
$$

for all $f, g \in L_{\omega_{\sigma}}^{1}(G)$, where $\check{\Delta}(y)=\Delta\left(y^{-1}\right)$. In particular, if $G$ is unimodular then the space $L_{\omega_{\sigma}}^{1}(G)$ is a convolution Banach algebra. 
Proof. That $L_{\omega_{\sigma}}^{1}(G)$ is a Banach space comes from weighted Lebesgue space theory; see for instance [7].

Observe that $\omega_{\sigma}(x)=\omega_{\sigma}\left(x y^{-1} y\right) \leq \omega_{\sigma}\left(x y^{-1}\right) \omega_{\sigma}(y)$. Then

$$
\begin{aligned}
\|f * g\|_{L_{\omega_{\sigma}}^{1}} & =\int_{G}\left|\int_{G} f\left(x y^{-1}\right) g(y) d y\right| \omega_{\sigma}(x) d x \\
& \leq \int_{G} \int_{G}\left|f\left(x y^{-1}\right)\right| \omega_{\sigma}\left(x y^{-1}\right)|g(y)| \omega_{\sigma}(y) d y d x \\
& \leq \int_{G} \int_{G}\left|f\left(x y^{-1}\right)\right| \omega_{\sigma}\left(x y^{-1}\right) d x|g(y)| \omega_{\sigma}(y) d y \\
& \leq \int_{G}|f(x)| \omega_{\sigma}(x) d x \int_{G} \Delta\left(y^{-1}\right)|g(y)| \omega_{\sigma}(y) d y \\
& \leq\|f\|_{L_{\omega_{\sigma}}^{1}}\|\check{\Delta} g\|_{L_{\omega_{\sigma}}^{1}}
\end{aligned}
$$

Now, if we assume that the group $G$ is unimodular, then $\Delta \equiv 1$. Therefore

$$
\|f * g\|_{L_{\omega_{\sigma}}^{1}} \leq\|f\|_{L_{\omega_{\sigma}}^{1}}\|g\|_{L_{\omega_{\sigma}}^{1}}
$$

Thus $L_{\omega_{\sigma}}^{1}(G)$ is a convolution Banach algebra.

\section{THE Fourier TRANSFORM}

For $f \in L_{\omega_{\sigma}}^{1}(G)$ we define the Fourier transform of $f$ by the formula

$$
\widehat{f}(\sigma)(T)=\int_{G}\left\langle\sigma(x)^{*}, T\right\rangle f(x) d x,
$$

where $T \in B_{1}\left(\mathfrak{H}_{\sigma}\right)$ and $\langle\cdot, \cdot\rangle$ denotes the duality bracket between $B_{1}\left(\mathfrak{H}_{\sigma}\right)$ and $B\left(\mathfrak{H}_{\sigma}\right)$.

Let us explain why the formula (8) is well-defined. By the boundedness of $\sigma(x)^{*}$, we have

$$
\left|\left\langle\sigma(x)^{*}, T\right\rangle\right| \leq\left\|\sigma(x)^{*}\right\|\|T\|_{1}=\|\sigma(x)\|\|T\|_{1} .
$$

Then,

$$
\int_{G}\left\langle\sigma(x)^{*}, T\right\rangle f(x) d x \leq\|T\|_{1} \int_{G}\|\sigma(x)\||f(x)| d x=\int_{G}|f(x)| \omega_{\sigma}(x) d x<\infty,
$$

since $f \in L_{\omega_{\sigma}}^{1}(G)$. This show the well-definedness of (8).

The formula (8) is quite a generalization of the classical Fourier transform. If $G=\mathbb{R}$ then from formula (8) one recovers the usual Fourier transform as it is explained in the proof of the following theorem.

Theorem 4.1. The formula (8) generalizes the Fourier transform formula in $\mathbb{R}$. 
Proof. Let $G=\mathbb{R}$. Then the (unitary irreducible) representation $\sigma$ is the character $\chi_{\omega}$ where $\chi_{\omega}$ is the function

$$
\chi_{\omega}: \mathbb{R} \longrightarrow \mathbb{T}, x \longmapsto e^{-i \omega x}
$$

One may identify $\chi_{\omega}$ with $\omega$. The representation space $\mathfrak{H}_{\sigma}$ is the set of complex numbers $\mathbb{C}$. The space of bounded operators $B\left(\mathfrak{H}_{\sigma}\right)$ and the space of trace-class operators $B_{1}\left(\mathfrak{H}_{\sigma}\right)$ are identical and are equal to the space of linear maps $L_{\alpha}: \mathbb{C} \longrightarrow \mathbb{C}, z \longmapsto \alpha z$ where $\alpha \in \mathbb{C}$. The invertible linear maps correspond to $\alpha \neq 0$. One may identify $L_{\alpha}$ with $\alpha$. Therefore if we apply (8) we obtain

$$
\widehat{f}(\omega) \alpha=\int_{\mathbb{R}}\left\langle\overline{\chi_{\omega}(x)}, \alpha\right\rangle f(x) d x=\int_{\mathbb{R}} \alpha e^{-i \omega x} f(x) d x .
$$

A simplification by $\alpha(\alpha \neq 0)$ gives

$$
\widehat{f}(\omega)=\int_{\mathbb{R}} e^{-i \omega x} f(x) d x
$$

which is the classical definition of the Fourier transform of $f$.

Remark 4.2. The fact that formula (8) generalizes the Fourier transform formula in locally compact abelian groups and compact non necessary abelian groups is straightforward since it can be obtain similarly form the above proof.

Let us define $L^{p}(G)$ to be the set of complex measurable (class of) functions $f$ on $G$ such that $\int_{G}|f(x)|^{p} d x<\infty, p \geq 1$. This set is endowed with the norm

$$
\|f\|_{L^{p}}=\left(\int_{G}|f(x)|^{p} d x\right)^{\frac{1}{p}} .
$$

Theorem 4.3. If $f \in L^{p}(G)$ and $\omega_{\sigma} \in L^{q}(G)$ with $\frac{1}{p}+\frac{1}{q}=1$, then $\widehat{f}(\sigma)$ is a bounded linear functional on $B_{1}\left(\mathfrak{H}_{\sigma}\right)$ and $\|\widehat{f}(\sigma)\| \leq\left\|\omega_{\sigma}\right\|_{L^{q}}\|f\|_{L^{p}}$.

Proof. Assume $f \in L^{p}(G)$ and $\omega_{\sigma} \in L^{q}(G)$. Then $f \omega_{\sigma} \in L^{1}(G)$. Therefore $f \in L_{\omega_{\sigma}}^{1}$. Now,

$$
|\widehat{f}(\sigma)(T)| \leq\|T\|_{1} \int_{G} \omega_{\sigma}(x)|f(x)| d x .
$$

Applying the Hölder inequality, we obtain

$$
|\widehat{f}(\sigma)(T)| \leq\|T\|_{1}\left(\int_{G} \omega_{\sigma}(x)^{q} d x\right)^{\frac{1}{q}}\left(\int_{G}|f(x)|^{p} d x\right)^{\frac{1}{p}} .
$$

That is $|\widehat{f}(\sigma)(T)| \leq\|T\|_{1}\left\|\omega_{\sigma}\right\|_{L^{q}}\|f\|_{L^{p}}$. Thus $\widehat{f}(\sigma)$ is bounded and $\|\widehat{f}(\sigma)\| \leq\left\|\omega_{\sigma}\right\|_{L^{q}}\|f\|_{L^{p}}$. 
Theorem 4.4. Assume that a sequence $\left(f_{n}\right)$ converges to $f$ in $L_{\omega_{\sigma}}^{1}(G)$. Then $\widehat{f}_{n}(\sigma)$ converges to $\widehat{f}(\sigma)$ in $B\left(\mathfrak{H}_{\sigma}\right)$.

Proof. From (8), the operator $\widehat{f}(\sigma)$ is given by

$$
\widehat{f}(\sigma)=\int_{G} \sigma(x)^{*} f(x) d x .
$$

Therefore,

$$
\begin{aligned}
\left\|\widehat{f}_{n}(\sigma)-\widehat{f}(\sigma)\right\| & =\left\|\int_{G} \sigma(x)^{*}\left(f_{n}(x)-f(x)\right) d x\right\| \\
& \leq \int_{G}\left\|\sigma(x)^{*}\right\|\left|f_{n}(x)-f(x)\right| d x \\
& \leq \int_{G}\|\sigma(x)\|\left|f_{n}(x)-f(x)\right| d x \\
& \leq \int_{G} \omega_{\sigma}(x)\left|f_{n}(x)-f(x)\right| d x \\
& \leq\left\|f_{n}-f\right\|_{L_{\omega_{\sigma}}^{1}} .
\end{aligned}
$$

Thus if $\left(f_{n}\right)$ converges to $f$ in $L_{\omega_{\sigma}}^{1}(G)$ then $\widehat{f}_{n}(\sigma)$ converges to $\widehat{f}(\sigma)$ in $B\left(\mathfrak{H}_{\sigma}\right)$. In other words, the mapping $f \longrightarrow \widehat{f}(\sigma)$ is a continuous (linear) operator from $L_{\omega_{\sigma}}^{1}(G)$ into $B\left(\mathfrak{H}_{\sigma}\right)$.

Theorem 4.5. Let $G$ be a compact group. If a sequence $\left(f_{n}\right)$ converges to $f$ in $L^{2}(G)$ then $\widehat{f}_{n}(\sigma)$ converges to $\widehat{f}(\sigma)$ in $B\left(\mathfrak{H}_{\sigma}\right)$.

Proof. Let $G$ be a compact group. Since the weight $\omega_{\sigma}$ is continuous on $G$ then $\omega_{\sigma}$ is bounded, that is $\left\|\omega_{\sigma}\right\|_{\infty}=\sup _{x \in G} \omega_{\sigma}(x)<\infty$. Now,

$$
\begin{aligned}
\int_{G}|f(x)| \omega_{\sigma}(x) d x & \leq\left\|\omega_{\sigma}\right\|_{\infty} \int_{G}|f(x)| d x \\
& \leq\left\|\omega_{\sigma}\right\|_{\infty}\left(\int_{G}|f(x)|^{2} d x\right)^{\frac{1}{2}} \sqrt{\mu(G)}
\end{aligned}
$$

where $\mu(G)$ stands for the finite Haar measure of $G$. We normalized this measure by setting $\mu(G)=1$. The last inequality shows that $L^{2}(G)$ is a subset of $L_{\omega_{\sigma}}^{1}(G)$. Therefore $\widehat{f}(\sigma)$ exists for $L^{2}(G)$-functions. Now,

$$
\begin{aligned}
\left\|\widehat{f}_{n}(\sigma)-\widehat{f}(\sigma)\right\| & =\left\|\int_{G} \sigma(x)^{*}\left(f_{n}(x)-f(x)\right) d x\right\| \\
& \leq\left\|\omega_{\sigma}\right\|_{\infty} \int_{G}\left|f_{n}(t)-f(t)\right| d t
\end{aligned}
$$


Now, we apply the Hölder inequality to obtain

$$
\begin{aligned}
\int_{G}\left|f_{n}(t)-f(t)\right| d t & \leq\left(\int_{G} 1^{2} d t\right)^{\frac{1}{2}}\left(\int_{G}\left|f_{n}(t)-f(t)\right|^{2} d t\right)^{\frac{1}{2}} \\
& \leq\left\|f_{n}-f\right\|_{2} .
\end{aligned}
$$

Therefore

$$
\left\|\widehat{f_{n}}(\sigma)-\widehat{f}(\sigma)\right\| \leq\left\|\omega_{\sigma}\right\|_{\infty}\left\|f_{n}-f\right\|_{2} .
$$

The conclusion follows.

\section{THE FOURIER TRANSFORM OF A SYSTEM}

In general, a Linear Time Invariant (LTI) System is a map $T: \mathscr{I} \longrightarrow \mathscr{O}$ where the space of inputs $\mathscr{I}$ and the space of outputs $\mathscr{O}$ are vector spaces which are closed under translation and the map $T$ is linear and commutes with translations [3]. We assume that there is a Fourier transformation $\mathscr{F}$ that maps functions in $\mathscr{I}$ to functions in $\mathscr{O}$ and that this Fourier transformation is an isomorphism. In this section, we aim to define the Fourier transform $\widehat{T}$ of the system $T$. If the input is the fonction $f$ then the output is a function $g$ defined by

$$
g=T f .
$$

If the equation (13) is carried in the frequency domain, then we obtain

$$
\mathscr{F} g=\widehat{T} \mathscr{F} f
$$

Therefore

$$
\mathscr{F} T f=\widehat{T} \mathscr{F} f .
$$

Finally, we obtain

$$
\widehat{T}=\mathscr{F} T \mathscr{F}^{-1} .
$$

Definition 5.1. Let $\mathscr{I}$ and $\mathscr{O}$ be respectively the set of inputs and the set of outputs. Assume that there is a bijective Fourier transformation from $\mathscr{I}$ onto $\mathscr{O}$. Let $T: \mathscr{I} \longrightarrow \mathscr{O}$ be a LTI operator. Then the Fourier transform of $T$ is given by

$$
\widehat{T}=\mathscr{F} T \mathscr{F}^{-1} .
$$


Theorem 5.2. (1) If $T$ is the convolution operator $T f=g * f$ then $\widehat{T}$ is the multiplication operator defined by $\widehat{T} f=\mathscr{F}(g) f$.

(2) If $T$ is the translation operator $(T f)(x)=f(x-a)$ then $\widehat{T}$ is the modulation operator defined by $(\widehat{T} f)(\omega)=e^{-i \omega a} f(\omega)$.

(3) If $\lambda$ is an eigenvalue of $T$ with eigenvector $f_{\lambda}$ then $\lambda$ is an eigenvalue of $\widehat{T}$ with eigenvector $\mathscr{F}\left(f_{\lambda}\right)$.

Proof. $\quad(1) \widehat{T} f=\mathscr{F} T \mathscr{F}^{-1} f=\mathscr{F}\left(g * \mathscr{F}^{-1} f\right)=\mathscr{F}(g) f$.

(2)

$$
\begin{aligned}
(\widehat{T} f)(\omega) & =\left(\mathscr{F} T \mathscr{F}^{-1}\right)(\omega) \\
& =\mathscr{F}\left(\tau_{a} \mathscr{F}^{-1} f\right)(\omega) \\
& =e^{-i \omega a} \mathscr{F}^{-1} f(\omega) \\
& =e^{-i \omega a} f(\omega) .
\end{aligned}
$$

$$
\begin{aligned}
\widehat{T} \mathscr{F}\left(f_{\lambda}\right) & =\mathscr{F} T \mathscr{F}^{-1} \mathscr{F}\left(f_{\lambda}\right) \\
& =\mathscr{F} T\left(f_{\lambda}\right)=\mathscr{F}\left(\lambda f_{\lambda}\right)=\lambda \mathscr{F}\left(f_{\lambda}\right) .
\end{aligned}
$$

\section{Conclusion}

We constructed a weighted Lebesgue-Banach convolution algebra. Then we defined a Fourier transform related to trace-class operators and a Fourier transform of a system. Convergence properties are obtained.

\section{REFERENCES}

[1] V.S.K. Assiamoua, A. Olubummo, Fourier-Stieltjes transforms of vector valued measures on compact groups, Acta Sci. Math. (Szeged) 53 (1989), 301-307.

[2] C. Bunpog, Multiplicative Fourier transform, Thai J. Math. 19 (2021), 113-124.

[3] M. Ciampa, M. Franciosi, M. Poletti, A note on impulse response for continuous, linear, time-invariant, continuous-time systems, IEEE Trans. Circuits Syst. I. 53 (2006), 106-113.

[4] G. B. Folland, A course in abstract harmonic analysis, CRC Press, Boca Raton, 1995.

[5] H. Führ, Abstract Harmonic Analysis of Continuous Wavelet Transforms, Lecture notes in Mathematics 1863, Springer-Verlag Berlin Heidelberg, 2005. 
[6] K. Gröchenig, Weight functions in time-frequency analysis. In: Rodino, L., Wong, M.-W. (eds.) Pseudodifferential Operators: Partial Differential Equations and Time-frequency Analysis, vol. 52, pp. 343-366. Fields Institute Comm. (2007).

[7] E. Kaniuth, A course in commutative Banach algebras, Springer, New York, 2009.

[8] Y. Mensah, Facts about the Fourier-Stieltjes transform of vector measures on compact groups, Int. J. Anal. Appl. 2 (2013), 19-25.

[9] V. S. Sunder, Operators on Hilbert space, Springer, New York, 2015.

[10] G. Warner, Harmonic analysis on semi-simple Lie groups I, Springer, New York, 1972 